\title{
Circulating tumor cells and neutrophil-lymphocyte ratio are predictive markers for metastatic colorectal cancer patients
}

\author{
Heming Li ${ }^{1,2 \#}$, Qiuge Liu ${ }^{1,2 \#}$, Shanshan Liang ${ }^{1,2 \#}$, Ping Yao ${ }^{1,2}$, Jinyan Lv ${ }^{1,2}$, Gang Wang ${ }^{1,2}$, Rongbin Tang ${ }^{1,2}$, \\ Tong Zhao ${ }^{1,2}$, Jiaoyang $\mathrm{Li}^{1,2}$, Lu Xu ${ }^{1,2}$, Lianli $\mathrm{Ma}^{1,2}$, Ruoyu Wang ${ }^{1,2}$ \\ ${ }^{1}$ Department of Oncology, Affiliated Zhongshan Hospital of Dalian University, Dalian, China; ${ }^{2}$ The Key Laboratory of Biomarker High Throughput \\ Screening and Target Translation of Breast and Gastrointestinal Tumor, Dalian, China \\ Contributions: (I) Conception and design: H Li, Q Liu, S Liang, R Wang; (II) Administrative support: P Yao; (III) Provision of study materials or \\ patients: J Lv, G Wang, R Tang; (IV) Collection and assembly of data: T Zhao, L Xu; (V) Data analysis and interpretation: L Ma; (VI) Manuscript \\ writing: All authors; (VII) Final approval of manuscript: All authors. \\ \#These authors contributed equally to this work. \\ Correspondence to: Ruoyu Wang. Department of Medical Oncology, Affiliated Zhongshan Hospital of Dalian University, No. 6 Jiefang Street, Dalian, \\ Liaoning 110006, China. Email: wangruoyu1963@hotmail.com.
}

\begin{abstract}
Background: More accurate predictive factors for colorectal cancer (CRC) are urgently needed. This study aimed to assess the potential prognostic roles of circulating tumor cells (CTCs), neutrophil-lymphocyte ratio (NLR), and platelet-lymphocyte ratio (PLR) in CRC patients.

Methods: Between 2014 and 2017, 118 CRC patients newly diagnosed at the Affiliated Zhongshan Hospital of Dalian University were retrospectively analyzed, including 72 (61\%) patients that underwent radical resection (resectable CRC) and 46 (39\%) advanced patients with metastatic CRC (mCRC). The CellSearch System was used to detect CTCs, and Spearman's correlation analyses tested the correlations between CTC counts and both NLR and PLR. Statistical analyses were performed using the Kaplan-Meier method, log-rank tests, and Cox proportional hazards models.
\end{abstract}

Results: Of the resectable cohort, $24 \%$ were positive for CTCs. Of the advanced cohort, $49 \%$ were positive for CTCs. The presence of CTCs was associated with advanced age ( $\geq 63$ years old; $\mathrm{P}=0.037$ ), a high PLR value $(\mathrm{P}=0.008)$, and a high NLR value $(\mathrm{P}=0.034)$. Additionally, baseline NLR [hazard ratio (HR) $=0.423 ; 95 \%$ confidence intervals $(\mathrm{CI}), 0.223-0.803 ; \mathrm{P}=0.008]$, PLR $(\mathrm{HR}=0.513 ; 95 \%$ CI, 0.276-0.954; $\mathrm{P}=0.035)$, and CTC counts (HR $=2.155 ; 95 \% \mathrm{CI}, 1.152-4.032 ; \mathrm{P}=0.016)$ were significantly associated with progression-free survival (PFS) in a univariate analysis of mCRC patients that received chemotherapy. Multivariate analysis further showed that NLR $(\mathrm{P}=0.044)$ and CTCs $(\mathrm{P}=0.047)$ were independent prognostic factors for mCRC patients.

Conclusions: This study provided evidence that NLR and CTC counts could serve as robust prognostic factors for patients with mCRC.

Keywords: Colorectal cancer (CRC); circulating tumor cells (CTCs); neutrophil-lymphocyte ratio (NLR); platelet-lymphocyte ratio (PLR); prognosis

Submitted May 08, 2020. Accepted for publication Sep 24, 2020.

doi: $10.21037 /$ tcr-20-2032

View this article at: http://dx.doi.org/10.21037/tcr-20-2032

^ ORCID: Heming Li: 0000-0002-5702-0147. 


\section{Introduction}

Colorectal cancer (CRC) is an increasingly common type of cancer; it is the second-leading cause of cancer-associated death worldwide $(1,2)$. Although much has been achieved in the past few decades in terms of early diagnosis and CTC treatment, many patients with advanced CRC still have a poor prognosis $(3,4)$. Recurrence and metastasis are significant problems that affect the long-term survival of patients with metastatic CRC (mCRC) $(5,6)$. Appropriate prognostic biomarkers are therefore urgently needed to predict both the risk of recurrence and patient prognosis. The biomarkers would provide clinicians with accurate information to guide their choice of appropriate therapeutic strategies for CRC patients.

Circulating tumor cells (CTCs) are often present in the blood of patients with solid tumors and travel via the bloodstream; CTCs are seeds for subsequent metastatic lesions in distant organs $(7,8)$. Increasing evidence suggests that baseline CTC counts and dynamic changes in CTC counts are independent predictors for patients with various types of cancer, including CRC (9). As CTC detection in peripheral blood is both feasible and reliable, CTCs have attracted the interest of researchers not only as a convenient tool with which to explore the underlying mechanisms of metastasis better but as clinically relevant prognostic markers for cancer patients.

Cancer development and inflammation are closely linked. Cancer patients commonly present with local and systemic changes in the immune-inflammation index, including changes in the neutrophil-lymphocyte ratio (NLR) and platelet-lymphocyte ratio (PLR) $(10,11)$. Many studies have shown that the immune-inflammation index is a powerful predictor of poor outcomes for CRC patients (12). Recent evidence has also shown that the interaction of inflammatory cells with CTCs in the peripheral blood may promote tumor metastasis to distant sites (13). Combining the immune-inflammation index and CTC detection as predictive tools may, therefore, allow for more accurate predictions of CRC patient survival.

This study aimed to assess the relationship between baseline CTC counts and general measures of the immuneinflammation index, namely, NLR and PLR. We further determined if baseline CTC counts in combination with NLR and PLR were associated with clinical outcomes in CRC patients. We present the following article in accordance with the STROBE reporting checklist (available at http://dx.doi.org/10.21037/tcr-20-2032).

\section{Methods}

\section{Patient selection}

Between 1 January 2014 and 31 December 2017, 118 patients that attended the Affiliated Zhongshan Hospital of Dalian University were enrolled in this retrospective study. Of these patients, 72 had resectable CRC and had undergone radical resection, and 46 had mCRC and had received first-line therapy. The study methodologies conformed to the provisions of the Declaration of Helsinki (as revised in 2013). This study was reviewed and approved by the Human Ethics Review Committee of the Affiliated Zhongshan Hospital of Dalian University (Protocol: 2020011; 2015032). All participants signed written informed consent. Of the 118 patients, 76 were male, and 42 were female. The age range was 36-89 years, and the median age was 63 years. Inclusion criteria were the following: (I) histologically confirmed CRC; and (II) adequate hematologic, hepatic, renal, and cardiac function. Exclusion criteria were the following: (I) patients who suffered perioperative or non-tumor-related death; (II) patients with severe bone marrow suppression following chemotherapy; (III) patients with autoimmune diseases, blood diseases, or infections; (IV) a history of other types of malignant tumors; (V) patients on long-term oral anticoagulants; and (VI) patients with severe cardiopulmonary diseases.

\section{Patient follow-up}

All patients were followed up at outpatient clinics and by telephone from the date of blood collection. A total of 4 patients who underwent radical resection were lost to follow-up; the rate of loss to follow-up was $3.39 \%$ (4/118). The follow-up deadline was 30 October 2018, and the median follow-up time was 25.4 months. The primary follow-up endpoint was disease-free survival (DFS) and progression-free survival (PFS). The participants' therapeutic response was assessed according to the American Joint Committee on Cancer (AJCC), the seventh edition.

\section{Data collection}

Peripheral blood samples were collected from all enrolled patients, either after radical resection (patients with resectable CRC) or before receiving first-line therapy (patients with $\mathrm{mCRC}$ ). At the time of admission, fasting venous blood was drawn: $7.5 \mathrm{~mL}$ for CTC detection using 
specialized CellSave storage tubes, and $5 \mathrm{~mL}$ for routine blood testing using BD Vacutainer EDTA-2K blood collection tubes.

\section{CTC detection}

CTC detection was performed using the CellSearch System, as described in previous literature (14). In brief, $6.5 \mathrm{~mL}$ of buffer was added to a specimen for dilution, then centrifuged at $800 \mathrm{~g}$ for $10 \mathrm{~min}$. It was then observed whether the plasma and red blood cells had separated in the sample tube. Following this, the supernatant was removed, magnetic beads conjugated with specific antibodies were added, and the samples were incubated for $20 \mathrm{~min}$ in a Magnest Cartridge. Fluorescently labeled with leukocytes marker (CD45) and epithelial cells marker (cytokeratin 8, 18, 19 and EpCAM) monoclonal antibodies were used to distinguish epithelial cells from leukocytes. CTCs were defined as nucleated cells with negative expression of CD45 and positive expression of cytokeratin and EpCAM. All magnetized specific antibodies not bound to CTCs were then washed off, and the enriched cells were re-enriched. Finally, cell fluorescence staining was performed using CellTracks Analyzer. Two professionals interpreted the results.

\section{NLR and PLR measurements}

Blood samples were collected, and routine laboratory analyses were performed, including total white blood cell, neutrophil, platelet, and lymphocyte counts. The NLR was defined as the neutrophil/lymphocyte ratio, and the PLR was defined as the platelet/lymphocyte ratio.

\section{Optimal cut-off value definitions}

The CTC positive standard and CTC definition criteria were determined by the following: the presence of cytokeratin antigen $(\mathrm{CK}+)$, tumor-specific nuclear antigen (DAPI+), and common leukocyte antigen (CD45-), as well as by manual counting. CTC $\geq 1$ was defined as CTC positive; and CTC $=0$ was defined as CTC negative. NLR $\geq 2.02$ was defined as a high NLR value; and NLR $<2.02$ was defined as a low NLR value. PLR $\geq 117.81$ was defined as a high PLR value; and PLR $<117.81$ was defined as a low PLR value. These were tested in strict accordance with the operating procedures. The testing environment complied with the relevant operating standards. The area assessed optimal cut-off points under the ROC curve (AUC).

\section{Statistical analysis}

Data processing and analysis were performed using SPSS 19.0 software (SPSS, Chicago, IL, USA). Continuous variables were shown as mean \pm standard deviation (SD) and categorical variables as percentages (\%). Spearman's correlation analysis evaluated correlations between CTC counts and both NLR and PLR. Associations between these markers and clinical parameters were evaluated using chisquare tests. The Kaplan-Meier method, the two-tailed log-rank test, and the Cox proportional hazard model were used to analyze survival. A P value of $<0.05$ was considered statistically significant.

\section{Results}

\section{Comparison of CTC counts, the immune-inflammation index and clinical features}

Between January 2014 and December 2017, 118 patients newly diagnosed with CRC at the Affiliated Zhongshan Hospital of Dalian University were enrolled in this study, including 72 (61\%) resectable CRC patients and 46 (39\%) mCRC patients. The follow-up deadline was 30 October 2018, and the median follow-up time was 25.4 months. Of the 118 participants, 4 resectable CRC patients were lost to follow-up, the rate of loss to follow-up being $3.39 \%$. The baseline variables tested were age, sex, tumor localization, and TNM (tumor, nodes, metastases) stage, metastatic sites, and therapeutic strategies. Of the resectable CRC cohort, 17 patients (24\%) were positive for CTCs, and 55 (76\%) were negative for CTCs (Table 1). Positive CTC status was significantly associated with stage III cancer $(\mathrm{P}=0.035)$ and $\mathrm{N}+$ status $(\mathrm{P}=0.025)$. There were no significant differences between CTC counts and measures of the immuneinflammation index, being NLR and PLR in the resectable cohort.

Of the mCRC cohort, $48 \%(22 / 46)$ were positive for CTCs, which was notably higher than in resectable patients. Additionally, positive CTC status was associated with advanced age ( $\geq 63$ years old; $P=0.037$ ), a high PLR value $(\mathrm{P}=0.008)$, and a high $\mathrm{NLR}$ value $(\mathrm{P}=0.034)$. However, CTC counts were not correlated with age, sex, tumor localization, metastatic sites, or therapeutic strategies (Table 2). 
Table 1 Relationship between CTCs counts and clinical parameters in 72 resectable CRC patients after radical resection

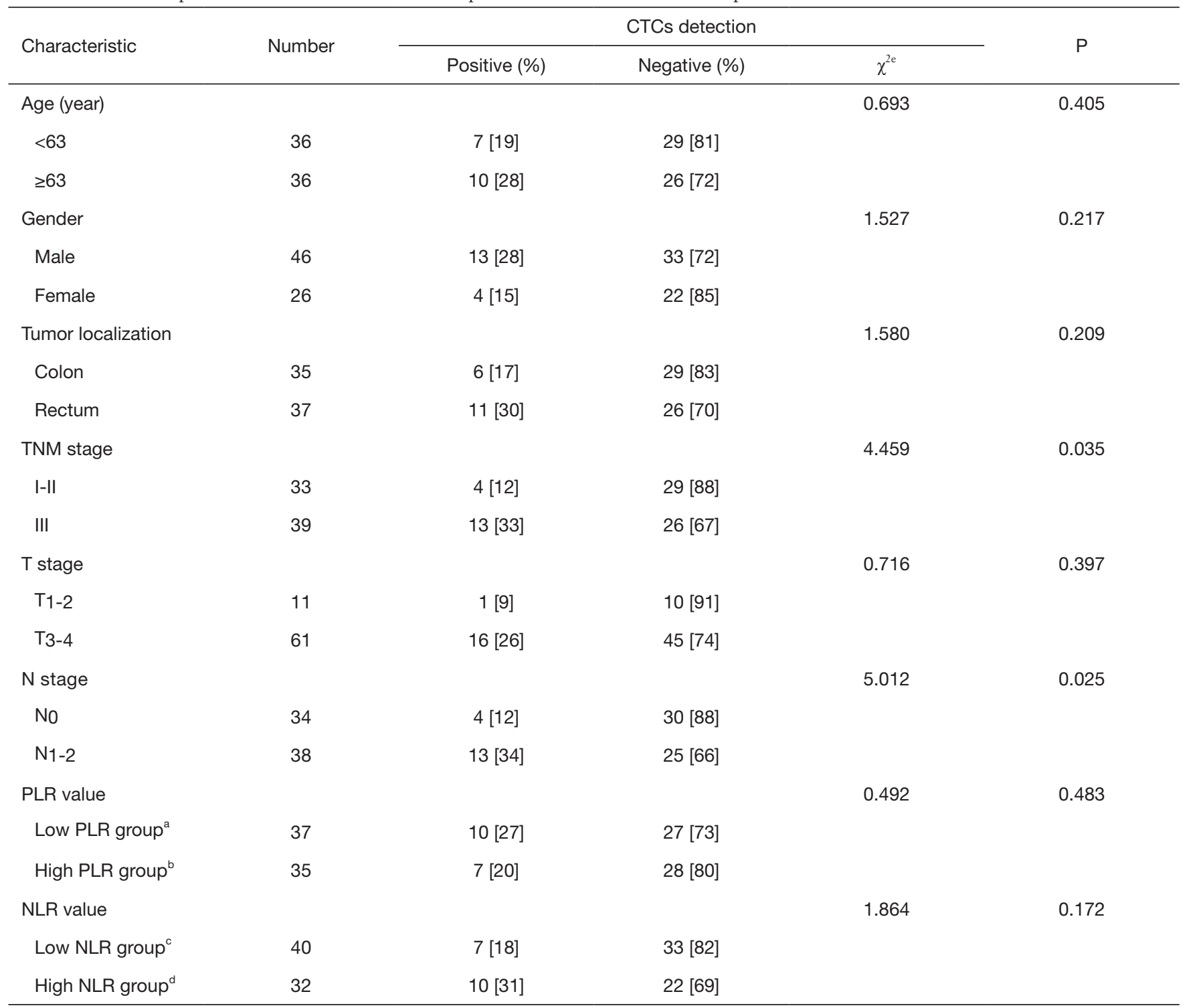

a, low PLR group, PLR <117.81; ${ }^{b}$, high PLR group, PLR $\geq 117.81 ;{ }^{c}$, low NLR group, NLR $<2.02 ;{ }^{d}$, high NLR group, NLR $\geq 2.02 ;{ }^{e}$,

Chi-square test. PLR, platelet-lymphocyte ratio; NLR, neutrophil-lymphocyte ratio; CTCs, circulating tumor cells.

\section{Relationship between CTC counts and the immune- inflammation index}

To assess the potential value of the immune-inflammation index in CTC detection, we further analyzed the correlation between CTC counts and two measures of the index, NLR and PLR. A significant positive correlation was found between CTC counts and PLR in the resectable CRC population $(\mathrm{P}=0.011)$ (Figure $1 A)$, though CTC counts were not positively correlated with NLR $(\mathrm{P}=0.079)$ (Figure 1B). However, in the mCRC cohort, CTC counts were positively correlated with both NLR $(\mathrm{P}=0.001)$ and PLR $(\mathrm{P}<0.001)$ (Figure $1 C, D)$.

\section{CTCs counts and prognosis of CRC patients}

Next, we assessed the prognostic value of baseline CTC counts in patients with CRC. The median follow-up time of the entire study population was 25.4 months. Of the resectable CRC patients, 15 of the $72(21 \%)$ relapsed or developed metastasis following radical resection. Positive 
Table 2 Correlation between baseline CTCs counts and clinical parameters in $46 \mathrm{mCRC}$ patients receiving first-line chemotherapy

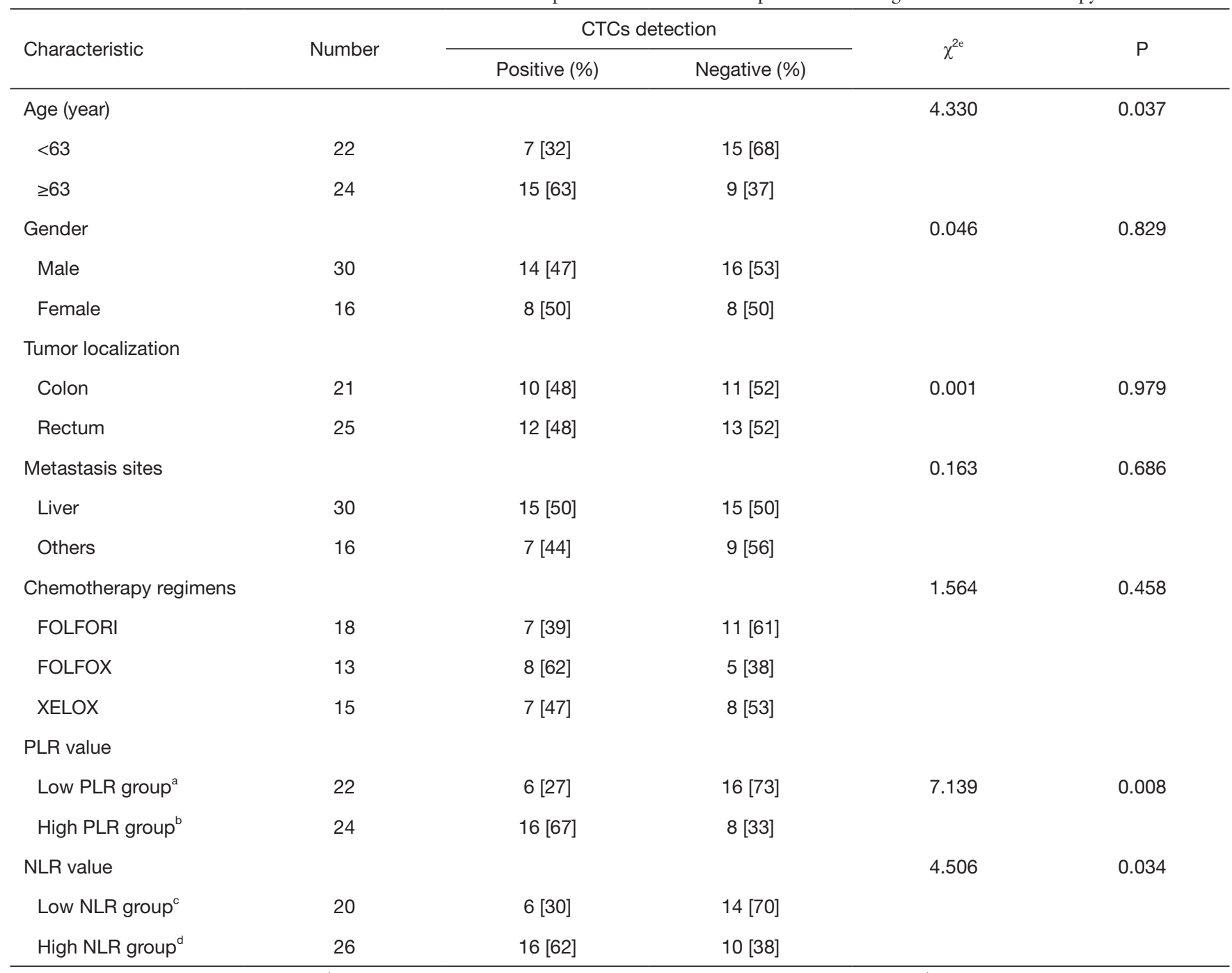

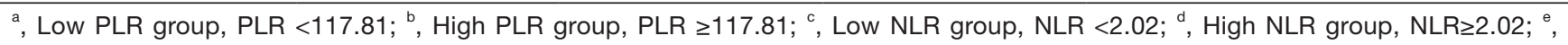
Chi-square test. PLR, platelet-lymphocyte ratio; NLR, neutrophil-lymphocyte ratio; CTCs, circulating tumor cells.

CTC detection was not associated with worse DFS in the resectable CRC cohort (CTC positive: $28 \pm 1.5$; CTC negative: $30 \pm 2.7$ months; $\mathrm{P}=0.258$ ) (Figure $2 A$ ). Of the mCRC patients, 43 of the $46(93 \%)$ had to disease progression following first-line therapy. There was a significant difference in PFS between the CTC positive and CTC negative mCRC patients (CTC positive: $2.5 \pm 0.3$ months; CTC negative: $6 \pm 0.5$ months; $\mathrm{P}=0.011$ ) (Figure 2B). This data suggests that CTC counts could predict whether mCRC patients show a therapeutic response to first-line therapy.

\section{Immune-inflammation index and prognosis of CRC patients}

We further evaluated the prognostic value of baseline NLR and PLR in CRC patients. The corresponding optimal cut-off values for NLR and PLR, as determined by ROC curves, were 2.02 and 117.81 , respectively. Participants were then separated into a high NLR group $(\geq 2.02)$, a low NLR group $(<2.02)$, a high PLR group $(\geq 117.81)$, and a low PLR group $(<117.81)$. For the resectable CRC cohort, there was no significant difference in DFS based on KaplanMeier curves stratified for either NLR $(\mathrm{P}=0.073)$ or PLR 
A

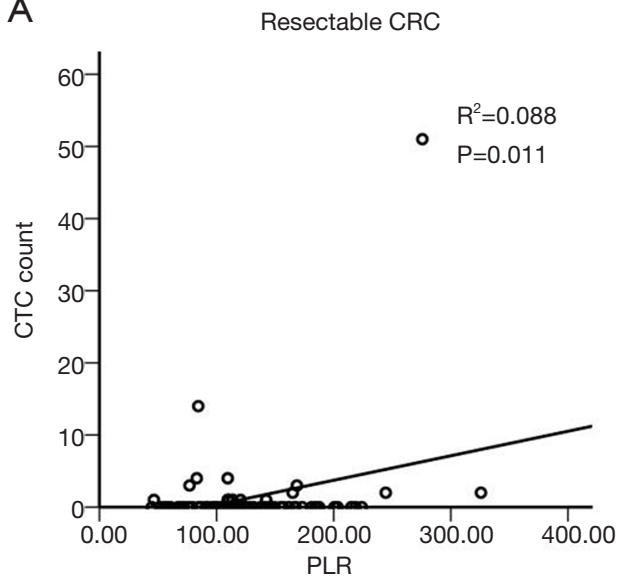

B

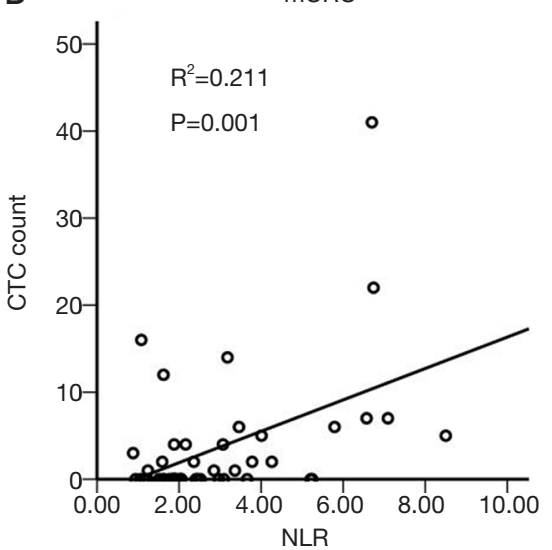

B

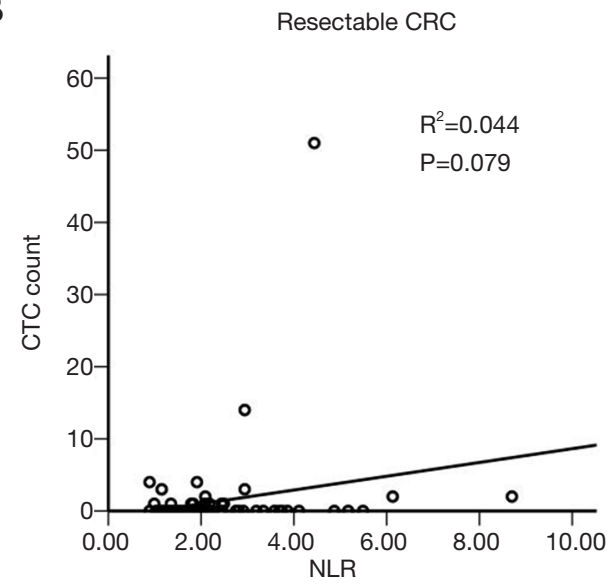

D

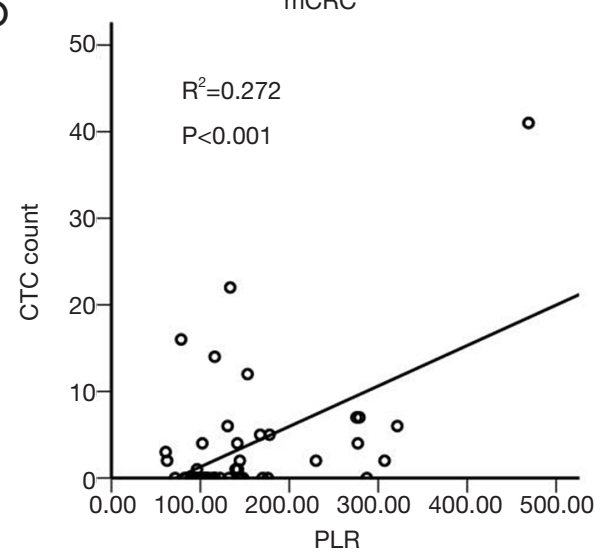

Figure 1 Spearman's correlation analysis between CTC counts and inflammation-based indexes. The scatter plots show the distribution of CTC counts and inflammation-based indexes in resectable CRC patients $(A, B)$ and mCRC patients (C,D). The connecting lines show the correlation of CTC counts with NLR and PLR. CTC, circulating tumor cell; CRC, colorectal cancer; mCRC, metastatic colorectal cancer; NLR, neutrophil lymphocyte ratio; PLR, platelet-lymphocyte ratio.
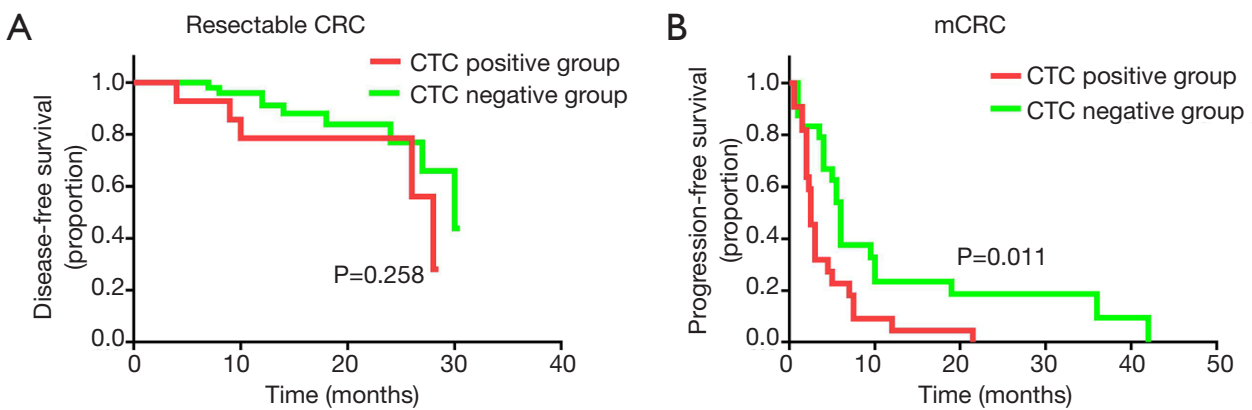

Figure 2 Kaplan-Meier curves for DFS and PFS according to baseline CTC counts. (A) DFS curves for resectable patients. (B) PFS curves for mCRC patients. The CTC negative patients had CTC counts $=0$; and the CTC positive patients had CTCs counts $\geq 1$. CTC, circulating tumor cell; mCRC, metastatic colorectal cancer; DFS, disease-free survival; PFS, progression-free survival. 
A

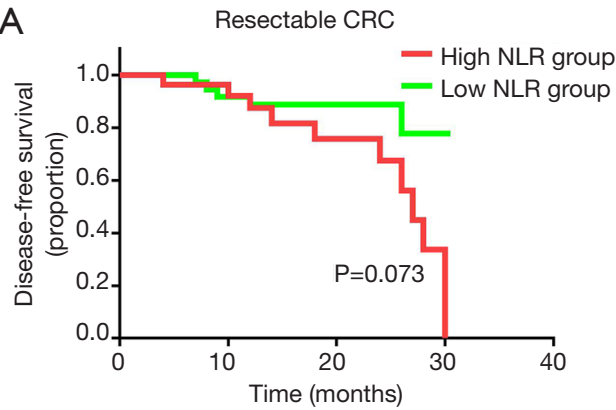

C

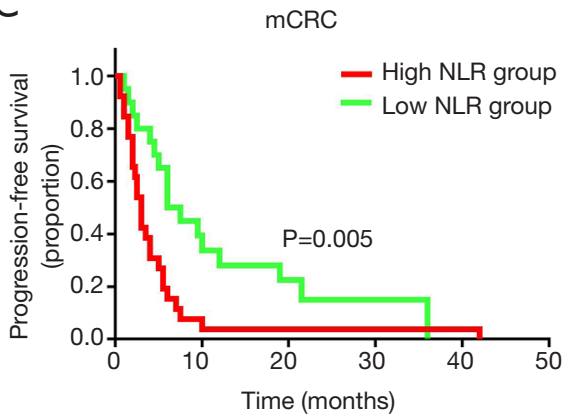

B
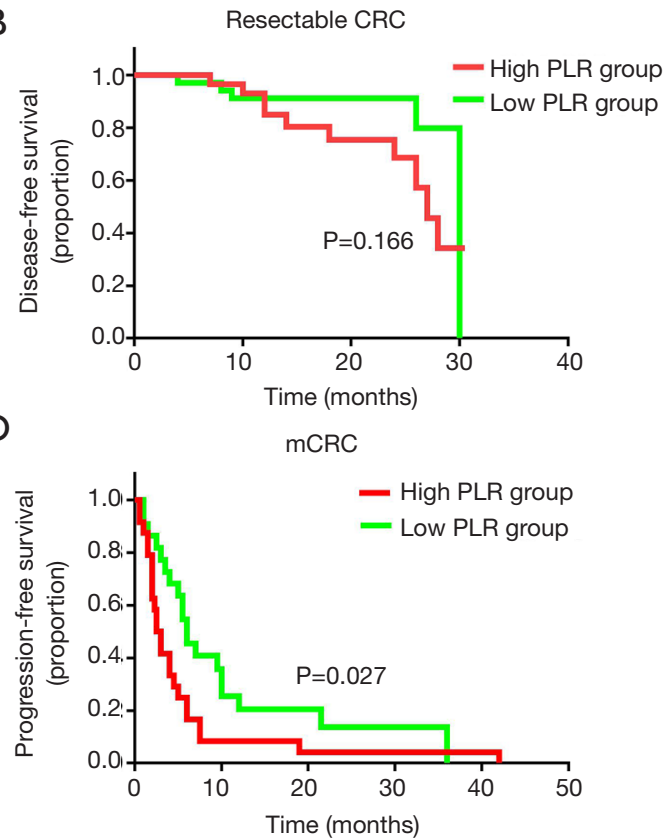

Figure 3 Kaplan-Meier curves for DFS and PFS according to baseline NLR and PLR. DFS curves for resectable patients grouped by (A) NLR and (B) PLR. PFS curves for mCRC patients grouped by (C) NLR and (D) PLR. For the high PLR value group, PLR $\geq 117.81$; for the low PLR value group, PLR <117.81; for the high NLR value group, NLR $\geq 2.02$; and for the low NLR value group, NLR <2.02. mCRC, metastatic colorectal cancer; DFS, disease-free survival; PFS, progression-free survival; NLR, Neutrophil lymphocyte ratio; PLR, Plateletlymphocyte ratio.

$(\mathrm{P}=0.166)$ (Figure $3 A, B)$. However, a worse PFS in mCRC patients was associated with high NLR values $(\mathrm{P}=0.005)$ and high PLR values $(\mathrm{P}=0.027)$ (Figure $3 C, D)$. These findings demonstrated that NLR and PLR are potential predictive markers for mCRC patient prognosis.

\section{Univariate and multivariate analyses of survival predictors}

We performed both univariate and multivariate analyses of survival outcomes for mCRC patients. In the univariate analysis, baseline NLR (HR $=0.423 ; 95 \%$ CI, 0.223-0.803; $\mathrm{P}=0.008)$, PLR (HR $=0.513 ; 95 \%$ CI, 0.276-0.954; $\mathrm{P}=0.035)$, and CTC counts (HR $=2.155$; 95\% CI, $1.152-$ 4.032; $\mathrm{P}=0.016)$ were significantly associated with PFS. Multivariate analysis of outcomes for mCRC patients further showed that low NLR values were independently associated with better PFS (HR $=0.503 ; 95 \%$ CI, $0.257-$ 0.981; $\mathrm{P}=0.044$ ) and higher CTC counts were significantly correlated with worse PFS (HR $=1.925$; 95\% CI, $1.008-$ 3.676; $\mathrm{P}=0.047)$. Although patients with higher PLR values appeared to have worse outcomes than those with lower PLR values, PLR was not an independent prognostic factor for the mCRC cohort $(\mathrm{P}=0.183)$ (Table 3). This data suggests that both NLR and CTC counts are independent risk factor for disease progression in $\mathrm{mCRC}$.

\section{Discussion}

Several clinical variables have been reported to predict survival outcomes of CRC patients, including TNM stage and performance status (PS) score. However, the prediction and assessment of relapse risk in patients with occult metastases using imaging techniques and serum tumor marker detection remain difficult. A convenient and dynamic method of predicting relapse risk and monitoring therapeutic responses are therefore urgently needed. Previous retrospective studies have shown that CTCs is an independent prognostic marker for CRC patients (15). Inflammation induces systemic changes within the cancer microenvironment that promote tumor progression, and CTCs may be directly involved in this inflammation process (16). Therefore, CTC detection used in combination with the immune-inflammation index may lead to more accurate predictions of therapeutic response and prognosis. In the 
Table 3 Univariate and multivariate analysis of PFS in patients with mCRC

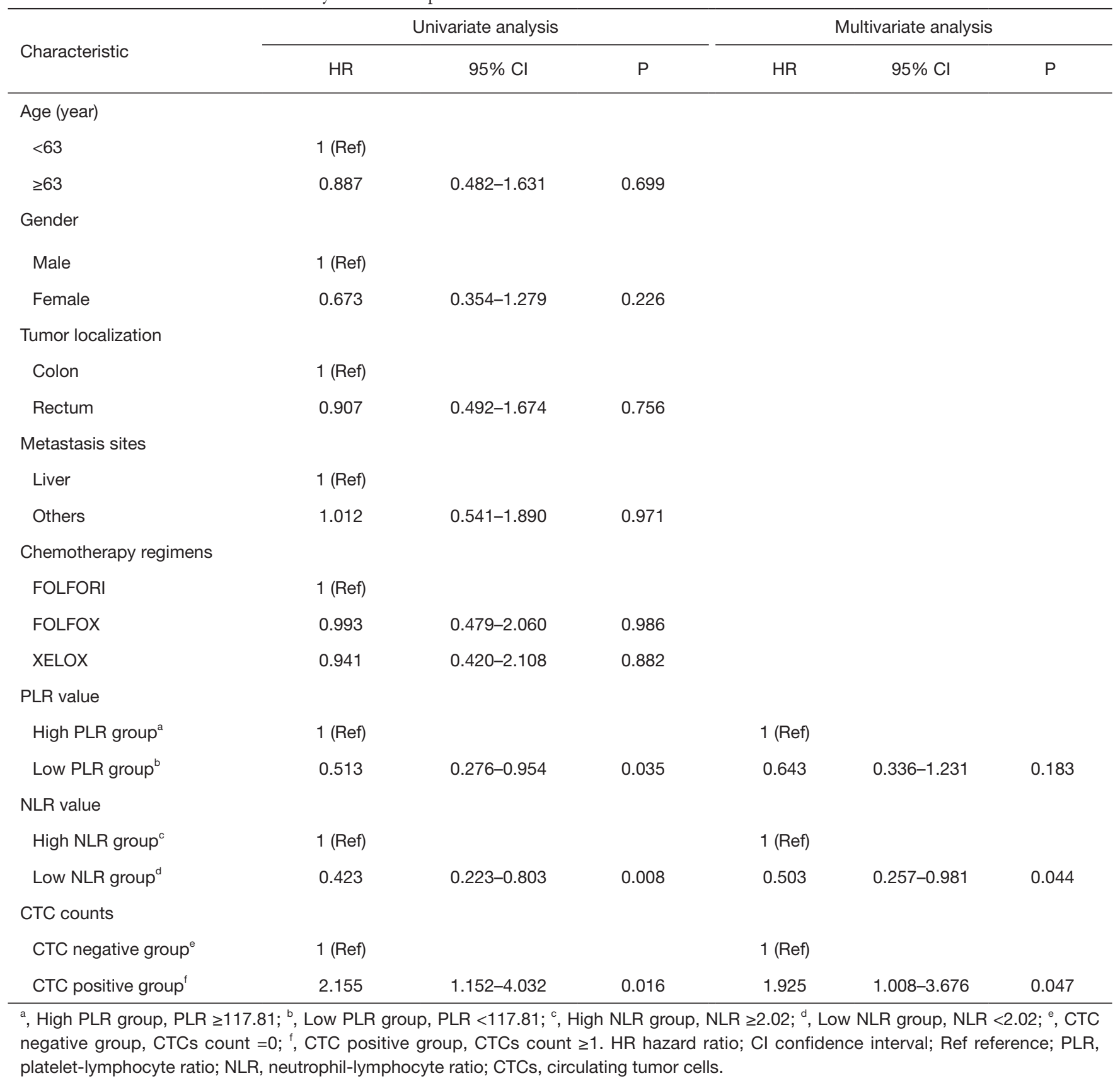

present study, we found for the first time that baseline CTC counts, together with NLR, were independent prognostic factors for patients with mCRC.

The molecular mechanisms by which CTC populations promote metastasis to a determined distant site are poorly understood. Theoretically, CTCs that leave the primary tumor site will be more vulnerable to attack by immune cells; the survival of CTCs in vessels might be the "Achilles' heel" of metastasis (17). Recent publications have shown that some CTCs closely interact with blood cells, such as neutrophils, platelets, and monocytes, to survive in the bloodstream $(13,18)$. It has been reported that neutrophils promote adhesion and metastases seeding of distant organ sites by secreting circulating growth factors, while lymphocytes play an essential role in tumor defense by inhibiting tumor cell proliferation and migration (19). Other 
studies have indicated that platelets induce the epithelialmesenchymal transition process of CTCs, promoting extravasation to metastatic sites (20). In the present study, we found that CTC counts were positively associated with the immune-inflammation index (as measured by NLR and PLR) in mCRC patients, and that baseline CTC counts, together with NLR, were independent prognostic factors for mCRC patients. These results are consistent with previous studies that investigated breast and gastric cancer. The interaction of CTCs with the blood microenvironment is crucial to the development of new metastatic sites, which makes these interactions a potential target for novel treatment strategies.

This study was affected by the limitations that come with retrospective studies. We found that baseline CTC counts, NLR, and PLR were potential predictive markers for mCRC patients, but not for resectable patients, and we found no evidence that mCRC patients could benefit from chemotherapy. These findings were not in agreement with other studies. Additionally, the CTC positive rate was $24 \%$ in the resectable cohort, and $49 \%$ in the mCRC cohort, which was lower than other studies have previously reported. There are three possible explanations for these discrepancies. Firstly, even though the sample size of this study was 118 , there were only 46 cases of mCRC (39\%), far fewer than those with resectable CRC (61\%). Secondly, our study was a retrospective single-center study. Finally, the participants had received a variety of treatments, including single chemotherapy treatment, chemotherapy and radiation, and other chemotherapy treatments combined with targeted therapy. These results may explain why we did not find that therapy regimens were significantly associated with PFS.

It is important to note that this study exhibited the several limitations of retrospective studies conducted at a single center with a smaller sample size. Moreover, in the subgroup analyses, it was impossible to obtain significant results due to the small sample size of this present study. Therefore, our findings require validation with large-scale, multicenter, controlled clinical studies.

\section{Conclusions}

CTC detection and NLR can be used as prognostic biomarkers for mCRC. CTC counts are significantly correlated with NLR and PLR, particularly in mCRC patients. The low cost, dynamic collection and ready accessibility to tools with which to assess CTC counts and
NLR make these promising prognostic factors for mCRC. The relationship between CTCs and NLR requires further investigation in more extensive clinical trials. Such trials may contribute to the development of new prognostic models for mCRC.

\section{Acknowledgments}

Funding: The study was supported by grants from Dalian Science and Technology Innovation Fund Project (Grant 2018J12SN063); Natural Science Foundation of Liaoning Province (Grant 2019-MS-010); Dalian Medical and Science Research Fund Project (Grant 1911096); Chinese Society of Clinical Oncology Research Funding (Grant Y-HR2018-117).

\section{Footnote}

Reporting Checklist: The authors have completed the STROBE reporting checklist. Available at http://dx.doi. org/10.21037/tcr-20-2032

Data Sharing Statement: Available at http://dx.doi. org/10.21037/tcr-20-2032

Peer Review File: Available at http://dx.doi.org/10.21037/tcr20-2032

Conflicts of Interest: All authors have completed the ICMJE uniform disclosure form (available at http://dx.doi. org/10.21037/tcr-20-2032). The authors have no conflicts of interest to declare.

Ethical Statement: The authors are accountable for all aspects of the work in ensuring that questions related to the accuracy or integrity of any part of the work are appropriately investigated and resolved. The study methodologies conformed to the provisions of the Declaration of Helsinki (as revised in 2013). This study was approved by the Ethics Committee of The Affiliated Zhongshan Hospital of Dalian University (Protocol: 2020011; 2015032). Signed written informed consent was obtained from the patient who was collected the blood sample.

Open Access Statement: This is an Open Access article distributed in accordance with the Creative Commons Attribution-NonCommercial-NoDerivs 4.0 International 
License (CC BY-NC-ND 4.0), which permits the noncommercial replication and distribution of the article with the strict proviso that no changes or edits are made and the original work is properly cited (including links to both the formal publication through the relevant DOI and the license). See: https://creativecommons.org/licenses/by-nc-nd/4.0/.

\section{References}

1. Segal RL, Miller KD, Jemal A. Cancer statistics, 2018. Ca Cancer J Clin 2018;68:7-30.

2. Ferlay J, Soerjomataram I, Dikshit R, et al. Cancer incidence and mortality worldwide: sources, methods and major patterns in GLOBOCAN 2012. Int J Cancer 2015;136:E359-86.

3. Bhullar DS, Barriuso J, Mullamitha S, et al. Biomarker concordance between primary colorectal cancer and its metastases. EBioMedicine 2019;40:363-74.

4. Cerdán-Santacruz C, Cano-Valderrama Ó, CárdenasCrespo S, et al. Colorectal cancer and its delayed diagnosis: have we improved in the past 25 years. Rev Esp Enferm Dig 2011;103:458-63.

5. Zippi M, De Toma G, Minervini G, et al. Desmoplasia influenced recurrence of disease and mortality in stage III colorectal cancer within five years after surgery and adjuvant therapy. Saudi J Gastroenterol 2017;23:39-44.

6. Anderson B, Fields RC. Prognosis and Management of Recurrent Metastatic Colorectal Cancer. Colorectal Cancer Liver Metastases. Berlin: Springer, 2020:571-87.

7. Rostami P, Kashaninejad N, Moshksayan K, et al. Novel approaches in cancer management with circulating tumor cell clusters. Journal of Science: Advanced Materials and Devices 2019;4:1-18.

8. Tellez-Gabriel M, Heymann MF, Heymann D. Circulating Tumor Cells as a Tool for Assessing Tumor Heterogeneity. Theranostics 2019;9:4580-94.

9. Wang D, Yang Y, Jin L, et al. Prognostic models based on postoperative circulating tumor cells can predict poor tumor recurrence-free survival in patients with stage II-III colorectal cancer. J Cancer 2019;10:4552-63.

10. Mollinedo F. Neutrophil degranulation, plasticity, and cancer metastasis. Trends Immunol 2019;40:228-42.

11. Zhang J, Zhang HY, Li J, et al. The elevated NLR, PLR and PLT may predict the prognosis of patients with colorectal cancer: a systematic review and meta-analysis. Oncotarget 2017;8:68837.

12. Chan JC, Chan DL, Diakos CI, et al. The lymphocyte-tomonocyte ratio is a superior predictor of overall survival in comparison to established biomarkers of resectable colorectal cancer. Ann Surg 2017;265:539-46.

13. Heeke S, Mograbi B, Alix-Panabières C, et al. Never travel alone: the crosstalk of circulating tumor cells and the blood microenvironment. Cells 2019;8:714.

14. Miller MC, Doyle GV, Terstappen LW. Significance of circulating tumor cells detected by the CellSearch system in patients with metastatic breast colorectal and prostate cancer. J Oncol 2010;2010:617421.

15. Hardingham JE, Grover P, Winter M, et al. Detection and clinical significance of circulating tumor cells in colorectal cancer-20 years of progress. Molecular Medicine 2015;21:S25-S31.

16. Quail DF, Joyce JA. Microenvironmental regulation of tumor progression and metastasis. Nat Med 2013;19:1423-37.

17. Mohme M, Riethdorf S, Pantel K. Circulating and disseminated tumour cells-mechanisms of immune surveillance and escape. Nat Rev Clin Oncol 2017;14:155-67.

18. Sprouse ML, Welte T, Boral D, et al. PMN-MDSCs Enhance CTC Metastatic Properties through Reciprocal Interactions via ROS/Notch/Nodal Signaling. Int J Mol Sci 2019;20:1916.

19. Szczerba BM, Castro-Giner F, Vetter M, et al. Neutrophils escort circulating tumour cells to enable cell cycle progression. Nature 2019;566:553-7.

20. Joosse SA, Pantel K. Tumor-educated platelets as liquid biopsy in cancer patients. Cancer cell 2015;28:552-4.
Cite this article as: Li H, Liu Q, Liang S, Yao P, Lv J, Wang G, Tang R, Zhao T, Li J, Xu L, Ma L, Wang R. Circulating tumor cells and neutrophil-lymphocyte ratio are predictive markers for metastatic colorectal cancer patients. Transl Cancer Res 2021;10(1):288-297. doi: 10.21037/tcr-20-2032 